Check for updates

1 HerpeZ, University Teaching Hospital, Lusaka, Zambia

2 Liverpool School of Tropical Medicine, Liverpool, UK

3 School of Life Sciences, University of Lincoln, Lincoln, UK

Correspondence to: J Tembo john.tembo@gmail.com Cite this as: $B M J \$$ \{year\};372:n457 http://dx.doi.org/10.1136/bmj.n457 Published:

\title{
Covid-19 in Africa
}

\section{Evidence of possible under-reporting of SARS-CoV-2 in Zambia}

John Tembo, ${ }^{1}$ Kwitaka Maluzi, ${ }^{1}, 2$ Franklyn Egbe, ${ }^{3}$ Matthew Bates ${ }^{1,3}$

In the latest World Health Organization epidemiological update (week 5, 2021 ${ }^{1}$ ), the number of cumulative cases of coronavirus disease 19 (covid-19) globally stands at 102 million, with 2.2 million deaths. During the first quarter of 2020, Africa was thought to be at high risk of covid-19 mortalities, with the WHO Office for Africa predicting up to 150 078 (best case scenario 82 735; worst case scenario 189 579) covid-19 related deaths in $2020 .{ }^{2}$ The most recent figures suggest that 62504 deaths with covid-19 have occurred in Africa. ${ }^{13}$ Various reasons for the muted impact of the pandemic in Africa have been postulated, including younger population demographics, rapid early quarantine measures, and pre-existing immunity. The relative contribution of these factors is unknown. ${ }^{4}$

Resource constrained settings need cost effective ways to track the pandemic and monitor the impact of public health interventions. Where clinical management of cases remains unchanged, covid-19 mortality roughly tracks disease incidence over time, so the linked study by Mwananyanda and colleagues (doi: 10.1136/bmj.n334), ${ }^{5}$ in which postmortem nasopharyngeal swabs were tested for severe acute respiratory syndrome coronavirus 2 (SARS-CoV-2), provides a crude but convenient way to track the progress of the pandemic in Zambia. This study and others like it are important, as they could help us to assess the impact of public health policy decisions.

Mwananyanda and colleagues screened postmortem nasopharyngeal swabs collected from $10 \%$ of all deaths reported at the University Teaching Hospital morgue in Lusaka, Zambia, between June and September 2020. They found $19.2 \%$ (70/364) to be positive for SARS-CoV-2 by reverse transcriptase polymerase chain reaction (RT-PCR). ${ }^{5}$ Most of the positive samples came from people who had died in the community and were not diagnosed as having covid-19 before death. Furthermore, only 32\% (6/19) of those who tested positive and who had died in hospital were tested before they died. A high proportion of positive cases were children $(10 \%$; 7/70).

Reported comorbidities among all deaths with covid-19 included tuberculosis (31\%; 22/70), hypertension $(27 \% ; 19 / 70)$, HIV/AIDS $(23 \% ; 16 / 70)$, alcohol misuse $(17 \% ; 12 / 70)$, and diabetes $(13 \%$; 9/70). This echoes findings from other studies, including one from the Western Cape in South Africa that found HIV, tuberculosis, diabetes, hypertension, and chronic kidney disease to be risk factors for death from covid-19. ${ }^{6}$ Mwananyanda and colleagues conclude that widespread community transmission of SARS-CoV-2 must be occurring in Zambia, and that many covid-19 cases and deaths are being missed or remain unreported. 5

Although their findings are compelling, the authors acknowledge that limitations exist as to how far their findings can be generalised, because sampling was done for only three to four months. The RT-PCR assay used was also changed during the study, which might have affected the analytical accuracy of the data, and they did not independently validate the assays before use.

It would be interesting to see how the data presented compare with national autopsy data on SARS-CoV-2 being collected by the Zambia National Public Health Institute (ZNPHI). ZNPHI is the body responsible for coordinating Zambia's pandemic response, and from the start of the pandemic its policy has required postmortem covid-19 testing for all deaths occurring inside or outside health facilities. Its most recent definition of a covid-19 death is any death following a clinically compatible illness, unless a clear alternative and unrelated cause of death can be ascertained. $^{7} 8$

Finally, although the prevalences of important comorbidities are reported, no odds ratios have been calculated to describe the degree to which these comorbidities might be associated with case category or RT-PCR results. The authors are doing further analyses to answer this question, and the findings will be of interest to help to inform the response to the pandemic in Zambia and elsewhere.

Mwananyanda and colleagues' findings add to the evidence that the impact of covid-19 in Zambia and elsewhere in Africa might be underestimated. Governments and funding bodies should fund comprehensive autopsy studies to characterise the range of pathologies now associated with covid-19 in Africa and to estimate the true effect of covid-19. ${ }^{9}$

Studies reporting accurate mortality rates in the years leading up to the pandemic are also needed, to help to quantify the number of excess deaths that may be attributable to covid-19 and to create a more accurate picture of the impact of this disease on the whole continent.

We acknowledge the support of the PANDORA network funded by the European and Developing Countries Clinical Trials Partnership (EDCTP).

Competing interests: We have read and understood BMJ policy on declaration of interests and declare the following interests: none.

Provenance and peer review: Commissioned; not peer reviewed.

World Health Organization. Weekly epidemiological update - 2 February 2021.2021. https://www.who.int/publications/m/item/weekly-epidemiological-update-2-february-2021 
2 Cabore JW, Karamagi HC, Kipruto H, etal. The potential effects of widespread community transmission of SARS-CoV-2 infection in the World Health Organization African Region: a predictive model. BMJ Glob Health2020;5:e0026475. doi: 10.1136/bmigh-2020-002647 pmid: 32451366

3 Pearson CA, Van Schalkwyk C, Foss AM, O'Reilly KM, Pulliam JRSACEMA Modelling and Analysis Response TeamCMMID COVID-19 working group. Projected early spread of COVID-19 in Africa through 1 June 2020. Euro Surveill 2020;25:2000543.

doi: 10.2807/1560-7917.ES.2020.25.18.2000543 pmid: 32400361

4 Musa HH, Musa TH, Musa IH, Musa IH, Ranciaro A, Campbell MC. Addressing Africa’s pandemic puzzle: Perspectives on COVID-19 transmission and mortality in sub-Saharan Africa. Int I Infect Dis 2021;102:483-8. doi: 10.1016/j.jij. 2020.09.1456 pmid: 33010461

5 Mwananyanda L, Gill C], MacLeod W, etal. Covid-19 deaths in Africa: prospective systematic postmortem surveillance study. BMJ 2021;372:n334.

6 Boulle A, Davies MA, Hussey H, etal. Risk factors for COVID-19 death in a population cohort study from the Western Cape Province, South Africa. Clin Infect Dis 2020;ciaa1198. doi: 10.1093/cid/ciaa1198 pmid: 32860699

7 Zambia National Public Health Institute. COVID-19 Intergrated Guidelines and Standard Operating Procedures. ZNPHI, 2020.

8 Zambia National Public Health Institute. The Revised (COVID-19) SARS-CoV-2 Testing Guidelines. ZNPHI, 2021.

9 Calabrese F, Pezzuto F, Fortarezza F, etal. Pulmonary pathology and COVID-19: lessons from autopsy. The experience of European Pulmonary Pathologists. Virchows Arch2020;477:359-72. doi: 10.1007/s00428-020-02886-6 pmid: 32642842

This article is made freely available for use in accordance with BMJ's website terms and conditions for the duration of the covid-19 pandemic or until otherwise determined by BMJ. You may use, download and print the article for any lawful, non-commercial purpose (including text and data mining) provided that all copyright notices and trade marks are retained. 